\title{
Die Rolle von lernenden Fabriken für Industrie 4.0
}

\author{
A. Kampker, C. Deutskens und A. Marks
}

Die Elektromobilität entwickelt sich momentan zu einer ernst zunehmenden Alternative zur herkömmlichen Fortbewegung durch Verbrennungsmotoren. Die Megatrends in der Gesellschaft - wie bspw. Neo-Ökologie und Mobilität - führen dazu, dass die Menschheit ihre Art sich fortzubewegen überdenkt. Sinkende Emissionsgrenzwerte und steigende Treibstoffpreise sind Beispiele aus dem Alltag, welche den Stellenwert der Elektromobilität erhöhen. Der Durchbruch auf dem Massenmarkt wird jedoch erst dann gelingen, wenn das Dilemma aus hohen Anschaffungskosten bei niedrigen Reichweiten aufgelöst werden kann. Sie lässt sich in diesem Zusammenhang als disruptive Innovation deklarieren. Aktuell weißt die Elektromobilität Eigenschaften einer disruptiven Innovation auf. Gemäß Christensen unterscheidet sie sich gegenüber erhaltenden Technologien vor allem im Hinblick auf das Wertesystem. Erhaltende Entwicklungen von Technologien finden in den Grenzen des bestehenden Wertesystems statt, also entlang der Dimensionen, die von den Kunden des Massenmarktes historisch zur Bewertung des Produktes herangezogen werden. Disruptive Technologien jedoch kennzeichnen sich durch die langfristige Veränderung des bestehenden Wertesystems. Während disruptive Technologien in der kurzen Frist zu einer Verschlechterung der Produkte verglichen mit den Produkten des Massenmarktes führen, sprechen sie durch spezielle Eigenschaften (neue Werte) Konsumenten in bestimmten Nischen an Christensen (1997). Sie können in den Nischenmärkten erfolgreich existieren, da deren Besetzung für die vorhandenen Marktmächte aufgrund des kleinen Volumens nicht interessant ist. Durch die dortige Marktbeteiligung gewinnt die Elektromobilität an Bedeutung, darüber hinaus werden dort wertvolle Erfahrungen gesammelt. Diese

Der Originaltext dieses Beitrags wurde überarbeitet. Das vollständige Korrekturverzeichnis finden Sie am Ende des Buchs und online unter http://dx.doi.org/10.1007/978-3-662-45915-7_16.

\footnotetext{
A. Kampker $(\varangle)$

StreetScooter GmbH, Jülicher Straße 191, 52070 Aachen, Deutschland

e-mail: A.Kampker@wzl.rwth-aachen.de

C. Deutskens · A. Marks

RWTH Aachen, WZL, Steinbachstr. 19, 52074 Aachen, Deutschland
} 
müssen genutzt werden, um die Technologie weiter zu entwickeln und dadurch bestehende Hemmnisse abzubauen, um sich damit den aktuellen Anforderungen des breiten Marktes anzunähern und diesen dann nachhaltig zu verändern.

\section{Maximierung des Return on Engineering}

Die technologische Reife der Elektromobilität ist Stand heute noch als sehr gering einzustufen, was neben der erwähnten Nischenanwendung kaum einen weiteren Absatzmarkt zulässt. Der durch Leistungsmerkmale begrenzte Nischenmarkt zeichnet sich durch eine hohe Individualität bei gleichzeitig niedrigen Stückzahlen und damit einer enormen Kostenherausforderung aus. Abbildung 1 stellt dies in den Dimensionen Kosten über Stückzahl exemplarisch dar. Die Marktlücke der kundenindividuellen und wirtschaftlichen Produktion wird aktuell nur durch automobile Kleinserien in Nischenmärkten, wie zum Beispiel Flottengeschäfte, möglich sein. Doch auch dann müssen die Anfangsinvestitionen gering gehalten werden und die Entwicklungsaufwände (in Zeit und Geld) begrenzt sein. Es wird damit der Ansatz verfolgt, die Vorteile des erhöhten Kundenwertes der Differenzierungsstrategie mit dem Vorteil der wirtschaftlichen Produktion der Kostenführerschaft zu vereinen. Zielgröße ist dabei der Return on Engineering (ROE), der den bereits erwähnten Quotienten aus erzieltem Nutzen zu investiertem Aufwand in allen Aktivitäten der Entwicklung und Produktion darstellt.

Insbesondere die kürzer werdenden Nachfragezyklen führen zu der Herausforderung, die Zeitspanne vom Startpunkt der Entwicklung bis zur Auslieferung des Fahrzeugs entsprechend zu minimieren. Hierfür sind entsprechende Kommunikationsinstrumente zu wählen und Prozesse zu definieren, durch welche Entwicklungsphasen parallel ablaufen können. Darüber hinaus sind möglichst modulare Produktstrukturen anzustreben, um durch eine geringe interne Varianz eine breite externe Varianz darstellen zu können, ohne einen großen Mehraufwand für Neuentwicklungen. Darüber hinaus muss es möglich sein, innerhalb kürzester Zeit den Produktionsanlauf zu bewältigen, was nur durch eine hohe
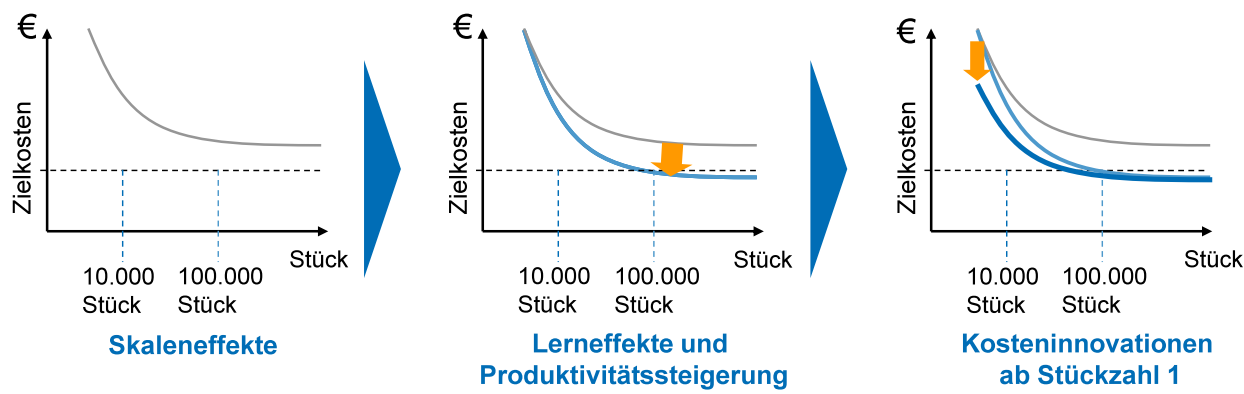

Reduzierung des Entwicklungsaufwands um die Hälfte der Zeit bei 1/10 der Investitionskosten RETURN ON ENGINEERING: ROE = T/2 + I/10

Abb. 1 Return on Engineering, Quelle: PEM der RWTH Aachen 
Flexibilität innerhalb der Montage erreicht werden kann. Daher liegt der anzustrebende Zielzustand bei der Hälfte des Zeitaufwands im Vergleich zu herkömmlichen Entwicklungszyklen aus der Automobilindustrie.

Über die verkürzte zeitliche Kapazität hinaus ist es - wie bereits erwähnt - notwendig, die Produktion und damit die hierfür notwendigen Investitionskosten entsprechend der Anforderungen und zu erwartenden Stückzahlen zu optimieren. Grundsätzlich neigt man in Hochlohnländern dazu, Flexibilität durch außerordentlich teure, hoch automatisierte Arbeitssysteme abzubilden, wobei diese dann auf hohe Stückzahlen ausgelegt sind. Die unsichere Stückzahlentwicklung sowie die beschriebene, gravierende Veränderung von Montagegegenstand und -Ablauf führen zu dem Bedarf nach einem skalierbaren, intelligenten Produktionssystem. Hierbei ist für die Investition in die Produktionsstruktur sowie den Anlauf als Zielwert ein Zehntel der konventionellen Ausgaben anzustreben.

\section{Die lernende Fabrik als Befähiger}

Die Elektromobilität und ihre starke technologische Weiterentwicklung erfordert Strukturen, die eine Einhaltung des eben beschriebenen Zielzustands ermöglichen. In der Struktur der Unternehmung ist ein technologisch gestützter, iterativer Verbesserungsprozess vorzusehen, um Weiterentwicklungen des Produkts zu ermöglichen, ohne die damit zusammenhängenden Kosten zu sprengen. Ferner ist ein Umfeld notwendig, welches die Anpassung von Mitarbeiter und Infrastruktur an die ständigen Veränderungen von Produkt und Prozess sowie dem Trend zu mehr Individualität in kürzerer Zeit ermöglicht.

Der bereits erwähnte Verbesserungsprozess lässt sich nur durch ein selbstoptimierendes, lernendes Produktionssystem durchlaufen. Das System und all seine Elemente müssen in der Lage sein, ihre Ziele bei veränderten Einflüssen anpassen zu können. Hierbei muss permanent ein dreistufiger Prozess der Selbstoptimierung durchlaufen werden. Der Analyse der Ist-Situation muss die Bestimmung der Ziele in Form von Auswahl, Anpassung oder Generierung eben dieser folgen. Abhängig davon sind in einem letzten Schritt entsprechende Anpassungen von Parametern, Strukturen oder des Verhaltens durchzuführen (Adelt et al. 2009). Im Hinblick auf die Entwicklungen im Rahmen der vierten industriellen Revolution, wird eine noch effizientere Nutzung durch ebendiese zunehmende Vernetzung in der Industrie möglich werden. Noch differenziertere, zeitaktuellere Daten werden durch die dezentrale, bedarfsgerechte Informationsbereitstellung in der lernenden Fabrik schnellere und zielgerichtete Optimierungen zulassen. Das lernende Produktionssystem ist daher als ein großer Profiteur der Industrie 4.0 anzusehen (Abb. 2).

Eine selbstständige Verbesserung und die daraus resultierenden Veränderungen - abhängig von den Einflussfaktoren - sind nur dann möglich, wenn physisch ein hohes Maß an Wandlungsfähigkeit gegeben ist. Das Potenzial hierzu lässt sich grundsätzlich anhand der acht Faktoren Universalität, Neutralität, Mobilität, Skalierbarkeit, Modularität, Kom- 


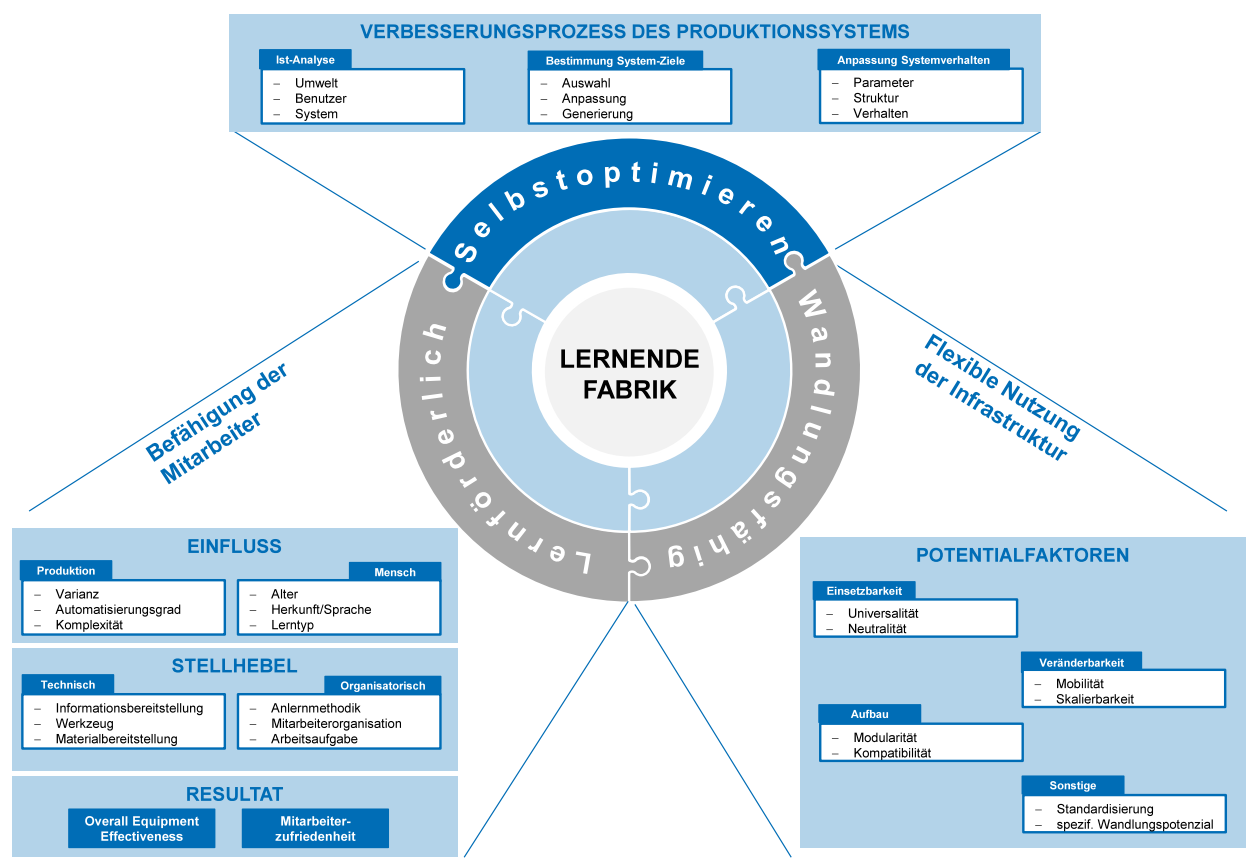

Abb. 2 Komponenten der lernenden Fabrik, Quelle: PEM der RWTH Aachen

patibilität, Standardisierung und objektspezifisches Wandlungspotenzial beschreiben (Heger 2007). Relevant für die weitere Betrachtung ist hierbei vor allem ein modularer und skalierbarer Aufbau der Produktion, welcher gleichzeitig universell genutzt werden kann und mobil ist bei Veränderungen. Die Vernetzung sämtlicher Bestandteile eines Produktionssystems führt zu einem hohen Bedarf an Flexibilität - in Bezug auf die physischen Elemente ist die Wandlungsfähigkeit einer Fabrik daher eine notwendige Voraussetzung für die Industrie 4.0.

Die Veränderung von Produkt, Prozess und Umfeld erschwert es dem Mitarbeiter als wesentlichem Produktionsfaktor schnell zu antizipieren und die geforderten Tätigkeiten in einer wirtschaftlichen Zeit durchzuführen. Die Lernförderlichkeit des Montagesystems ist aus diesem Grunde zwingend notwendig. Man versteht unter ihr die technische (Informationsbereitstellung, etc.) und organisatorische (Anlernmethodiken, etc.) Gestaltung eines Systems, welche den unbeeinflussbaren Faktoren - resultierend aus Produktion und Mensch - entsprechend durchzuführen ist. Je höher die Gesamtanlagenverfügbarkeit des Montagesystems ist und die Mitarbeiter zufrieden sind, desto mehr ist diese erfüllt. Für die aktuelle industrielle Revolution ist Sie das Pendant zur Wandlungsfähigkeit, um auch die psychische Flexibilität des Produktionsfaktor Mensch zu gewährleisten. 
Durch die Erfüllung dieser drei Eigenschaften wird aus einem normalen Produktionsumfeld eine Umgebung, die eine permanente Weiterentwicklung ermöglicht - die lernende Fabrik. Nur damit ist eine erfolgreiche Bearbeitung eines kleinvolumigen, volatilen Marktes mit geringem technologischen Reifegrad möglich.

\section{Das ZEP als Beispiel für eine lernende Fabrik}

Neu gegründeten, kleinen und mittelständischen Unternehmen fehlt es häufig an den monetären und zeitlichen Kapazitäten, um sowohl eine unreife Technologie weiter entwickeln, als auch entsprechende Produktionskonzepte einsetzen zu können, mit deren Hilfe unterschiedliche Entwicklungsstände kurz hintereinander auf den Markt gebracht werden. Für eine erfolgreiche Etablierung der Elektromobilität wurde durch das Zentrum für Elektromobilproduktion (ZEP) der zwingend erforderliche Schulterschluss zwischen Forschung und Industrie vollzogen. Innerhalb dieses Netzwerks wird Produktionsforschung betrieben, um auf der einen Seite die Bezahlbarkeit von E-Mobilität zu erreichen und gleichzeitig den Markt mit technologisch reifen Lösungen versorgen zu können. Durch die realitätsnahen Bedingungen wird ferner eine nahezu nahtlose Übertragung in die Praxis ermöglicht. Der gesamte Produktzyklus bis zur Serienproduktion, von der Entwicklung des Produkts bis zur Validierung von Fertigungsprozessen und Serienanläufen, wird durch die Entitäten Elektromobilitätslabor (eLab), Anlauffabrik und Demonstrationsfabrik (DFA) erforscht. Der StreetScooter transformiert diese Ergebnisse in die Praxis, worauf später noch genauer eingegangen wird (Abb. 3).

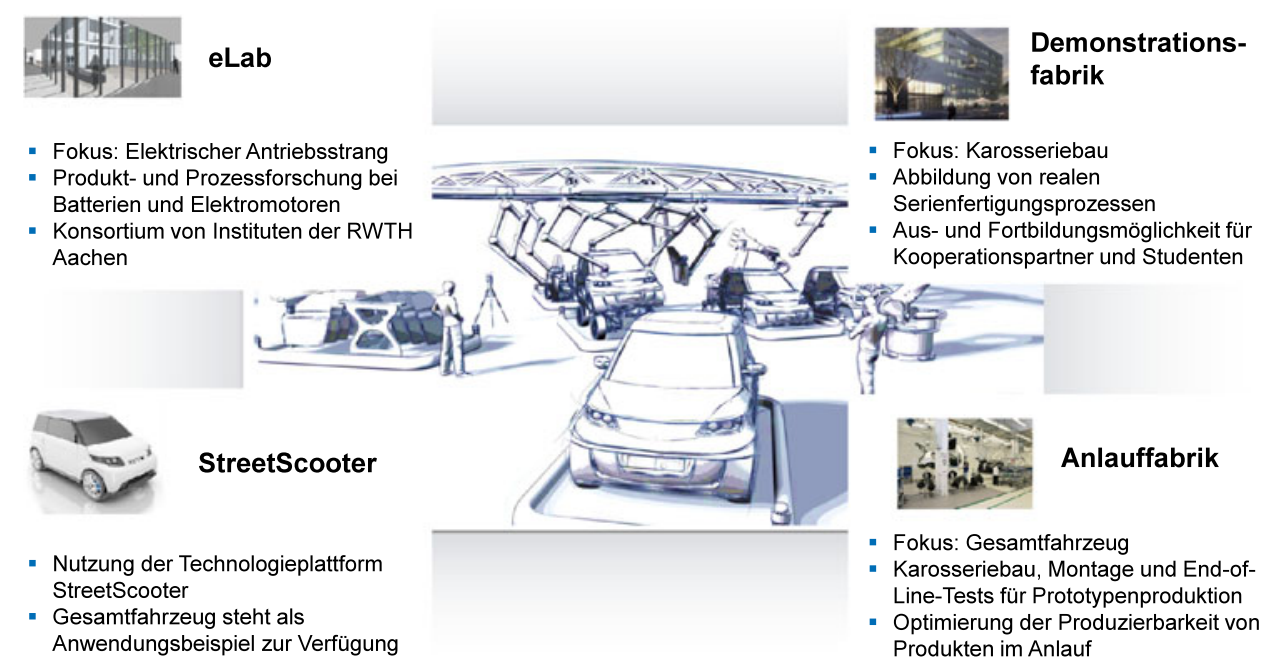

Abb. 3 Struktur des Zentrums für Elektromobilproduktion, Quelle: PEM der RWTH Aachen 
Das eLab konzentriert sich hierbei auf die Entwicklung neuer Antriebskonzepte und soll als Grundstein für die wirtschaftliche Serienproduktion von Elektrofahrzeugen dienen. Um höhere Leistung bei niedrigeren Preisen in Zukunft gewährleisten zu können, wird hierbei ein ganzheitlicher Entwicklungsansatz verfolgt. Dieser forschungsintensive Bereich wird durch die Integration verschiedenster Ingenieursdisziplinen bearbeitet. Es dient als Basis für die Entwicklung, Optimierung und Qualität von Komponenten und Prototypen für den elektrischen Antriebsstrang. Möglich ist dieser integrative Ansatz nur durch die entsprechende (informations-) technische und strukturelle Plattform. Die einzelnen Komponenten und ihre Produktionsprozesse werden hierbei permanent analysiert und die dabei entstehenden Daten an die entsprechenden Stellen transportiert. Der Analyse folgen bei Bedarf die Definition neuer Leistungsziele, welche dann durch entsprechende Veränderung von Prozessparametern oder -strukturen auch erreicht werden. Es ist ein Paradebeispiel für ein selbstoptimierendes, lernendes System und bringt die Elektromobilität dadurch in großen Schritten voran.

Die Anlauffabrik als nächster Schritt entlang des Produktlebenszyklus eines Elektrofahrzeugs untersucht die Herausforderungen, welche sich beim Serienanlauf durch die geringe vorhandene Erfahrung bzgl. Elektromobilität bei gleichzeitig hochkomplexen Prozessen ergeben. Im Fokus steht hierbei die Produzierbarkeit, wobei unter serienähnlichen Bedingungen die Prozesse auf die Probe gestellt werden, um später eine Massenproduktfähigkeit der entwickelten Produkt- und Produktionskonzepte gewährleisten zu können. Karosseriebau bei neuartigen Materialkombinationen, Fahrzeugmontage kritischer Komponenten wie bspw. das Battery Pack oder das End-of-Line-Testing technologisch neuer Fahrzeuge bilden wesentliche Problemfelder der E-Fahrzeug-Produktion und werden mit dem Ziel „Serienreife“ erforscht. Erkenntnisse in Bezug auf Produkt und Prozess werden an die notwendigen Stellen geliefert, woraus Verbesserungen resultieren sollen, welche dann zeitnah umgesetzt werden können. Hierfür wird, neben der entsprechenden technischen Infrastruktur zur Selbstoptimierung, durch den modularen Aufbau der Produktion und die Skalierbarkeit in Form von Erweiterungsmöglichkeiten, eine hohe Wandlungsfähigkeit gewährleistet. Ferner werden lernförderliche Elemente unter den Prämissen Produkt-/Stückzahlvarianz und begrenzter Vorqualifikation der Mitarbeiter eingesetzt und mit dem Ziel weiterentwickelt, hohe Produktivität in kurzer Zeit zu erreichen.

Die DFA als weiteres Kompetenzzentrum dient der Erprobung, Validierung und Weiterentwicklung von Produktionskonzepten für die Kleinserie. Durch die Produktion von Prototypen und fertig entwickelten Produkten liefert sie neben der Elektromobilität auch jeglichen anderen Unternehmen die Möglichkeit, im Vorfeld der eigenen Serienproduktion die Planung unter realen Bedingungen zu simulieren. Daher ist, neben der Informationsrückkopplung eine universelle und mobile Produktion vorhanden, welche die Erprobung sowohl unterschiedlicher Produkte als auch optimierter Abläufe zulässt. Ein wesentlicher Bestandteil neben der experimentellen Produktion und der Forschung ist hierbei jedoch auch die Weiterbildung von Mitarbeiten. Durch die Erprobung und dem damit verbundenen Einsatz aktuellster Erkenntnisse hinsichtlich der Gestaltung lernförderlicher Arbeitssysteme liefert Sie die ideale Plattform, um Mitarbeiter im Vorfeld der Produktion eigener 
Produkte extern zu qualifizieren. Ferner wird durch die Vernetzung von der Maschinenbis zur Fabrikebene eine permanente Überwachung in Echtzeit ermöglicht.

Zusammenfassend sind diese drei Entitäten das wichtige Bindeglied zwischen Forschung und Industrie, welches den Durchbruch der Elektromobilität beschleunigen wird. Erkenntnisse aus der Wissenschaft werden unter realen Bedingungen validiert und industrialisiert, wobei der herausragende Mehrwert hierbei die unmittelbaren Wechselwirkungen mit Unternehmen aus der Praxis sind.

\section{StreetScooter - Lernen in und aus der Praxis}

Der Grund für die Gründung des Unternehmens StreetScooter im Jahre 2011 war die Überzeugung, dass ein realer Bedarf nach Elektromobilität in bestimmten Anforderungsklassen existiert. Das Zusammenspiel der neuesten Erkenntnisse aus der Forschung, gepaart mit fachfremden, in ihrer Disziplin jedoch erfahrenen Unternehmen, haben zum erfolgreichen Gelingen des Vorhabens geführt. Als weiterer Bestandteil des ZEP kommt StreetScooter eine Doppelrolle zu - zum einen fungiert das Unternehmen als Ohr zum Markt bzw. dessen Anforderungen. Auf der anderen Seite dient es der Anwendung neuer Konzepte und Forschungsergebnisse in der Praxis und ist damit als Technologieplattform anzusehen. Die tatsächliche Produktion der Fahrzeuge, außerhalb des ZEP, nutzt die drei oben beschriebenen Säulen der lernenden Fabrik, angepasst auf die spezifischen Bedarfe.

Das Herzstück der lernenden Fabrik, ein sich selbst optimierendes, lernendes Produktionssystem, findet sich hier leicht abgewandelt wieder. Das heterarchische Entwicklungsnetzwerk ermöglicht allen Beteiligten die Analyse der einzelnen Komponenten sowie die Möglichkeit, Verbesserungen an die richtige Stelle zu adressieren. Nach Überprüfung durch die Verantwortlichen ist eine Optimierung im Sinne der Gesamtheit durch Anpassung gewisser Spezifikationen möglich. Das gleichberechtigte Netzwerk generiert durch wenig restriktive Produktspezifikationen und Kommunikation eine optimale KnowHow- und Innovationsausschöpfung. Entgegen der herkömmlichen, hierarchischen Lieferantenstrukturen wird den Unternehmen hier ein hohes Maß an Eigenständigkeit ermöglicht und damit ein unmittelbarer Austausch von Informationen, deren Interpretation und eine zeitaktuelle Umsetzung ermöglicht. Hauptelemente dieses Ansatzes sind die kooperative und vertrauensvolle Beziehung zwischen den Netzwerkpartnern, deren Heterogenität und eine systemunterstützte Kommunikation durch ein integratives Product-Life-CycleManagement.

Die Wandlungsfähigkeit als dringend notwendige Eigenschaft für die Produktion und damit das Unternehmen, welches sich in einem kundenindividuellen, von kleinen Stückzahlen geprägten Markt befindet, wird durch einen effizienten Entwicklungsprozess unterstützt. Value Engineering rückt hierbei den Kundenwert in den Mittelpunkt und bindet den zukünftigen Abnehmer früh in die Produktentstehung ein. So wird die häufig auftretende Unwissenheit der Unternehmen bzgl. der Kundenanforderungen und das daraus resultierende Over-Engineering vermieden. Durch den frühen Abgleich zwischen den internen 


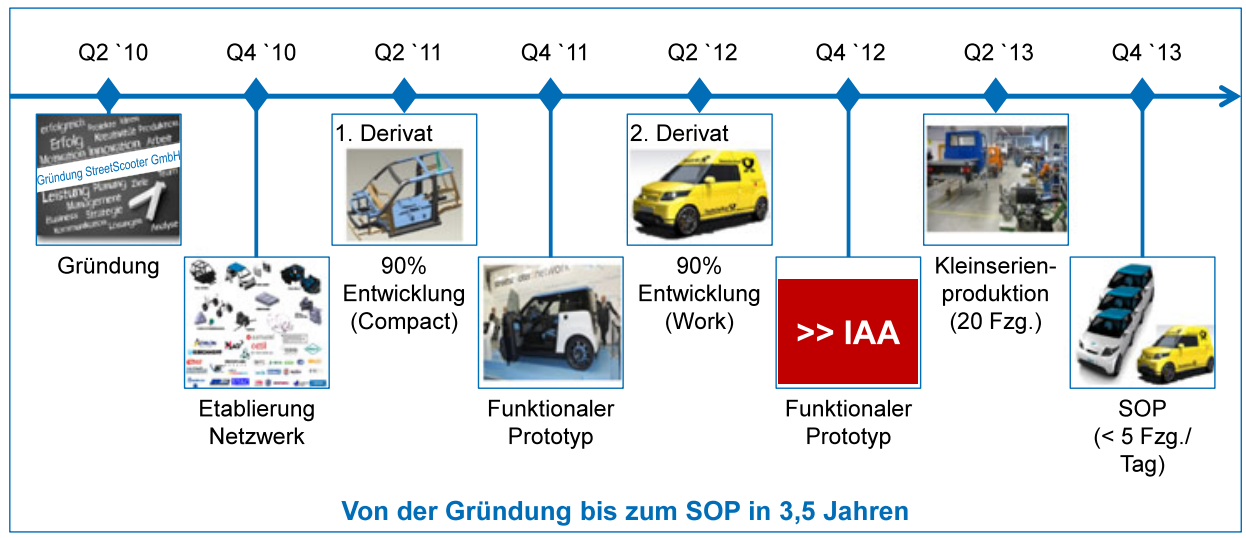

Abb. 4 Meilensteine des Projektes StreetScooter, Quelle: StreetScooter GmbH

Gegebenheiten mit den individuellen Wünschen und Restriktionen des Kunden ist eine effizientere Nutzung bestehender Infrastruktur möglich.

Die Varianz an Stückzahlen und Derivaten erfordert eine schnelle Adaption der Mitarbeiter an deren auszuführenden Montagetätigkeiten. Bei StreetScooter ist die Technologieplattform ein wesentlicher Befähiger für die entsprechende Prozessgestaltung (Abb. 4). Die integrierte Produkt- und Prozessentwicklung sowie die parallele Erarbeitung von Produkt- und Prozessbaukästen führen zu einer Minimierung der internen Komplexität. Durch die Baukastensystematik kommen hierbei eine begrenzte Anzahl an Montagekonzepten und Produktlösungen zum Einsatz. Die in Bezug auf die Lernförderlichkeit wichtigen, variantenunabhängigen Bereitstellungsarten von Werkzeug und Materialien, sowie die jederzeit und überall verfügbare Informationen und Anweisungen, erleichtern und fördern die lernförderliche Gestaltung. Die Mitarbeiter erhalten sich so ein gewisses Produktivitätsniveau und erreichen schneller den Zielzustand für das jeweilige Produkt wieder.

Der Beweis dafür, dass die beschriebene lernende Fabrik der Befähiger ist, um in kürzester Zeit und durch geringe Investitionen kundenindividuelle Serien fertigen zu können, ist der StreetScooter Carrier. In nur 3,5 Jahren zwischen Gründung und dem Start der Serienproduktion wurde ein Fahrzeug auf die Beine gestellt, von dem nun 50 Fahrzeuge im Dienste der Post tagtäglich Briefe und Pakete innerhalb Deutschlands zustellen. Das Flottengeschäft ist die Nische, welche der Elektromobilität zum Durchbruch verhelfen wird. Im Rahmen der kundenindividuellen Serienfertigung für E-Fahrzeuge könnten beispielsweise Pflegedienste ein möglicher nächster Absatzmarkt sein. Durch den Megatrend der Urbanisierung und die dadurch geringer werdenden Distanzen, bei einer gleichzeitig dichteren Infrastruktur von Aufladestationen, werden potenziell den Einstieg der Elektromobilität in den Massenmarkt ermöglichen. 
Open Access This chapter is distributed under the terms of the Creative Commons Attribution Noncommercial License, which permits any noncommercial use, distribution, and reproduction in any medium, provided the original author(s) and source are credited.

\section{Literaturverzeichnis}

Christensen, C. M. (1997). The innovator's dilemma - when new technologies cause great firms to fail (Microsoft Reader edition) (S. 11). Boston: Harvard Business School Press.

Adelt, P., Donoth, J., Geisler, J., Henkler, S., Kahl, S., Klöpper, B., Krupp, A., Münch, E., Paiz, C., Romaus, C., Schmidt, A., Schulz, B., Tscheuschner, T., Vöcking, H., Witkowski, U., Znamenshchykov, O., Oberthuer, S., Witting, K., Stöcklein, J., \& Porrmann, M. (2009). Selbstoptimierende Systeme des Maschinenbaus - Definitionen, Anwendungen, Konzepte. In J. Gausemeier, F. J. Rammig, \& W. Schäfer (Hrsg.), Selbstoptimierende Systeme des Maschinenbaus (S. 18-28). Paderborn: W.V. Westfalia Druck.

Heger, C. L. (2007). Bewertung der Wandlungsfähigkeit von Fabriken. In P. Nyhuis (Hrsg.), Berichte aus dem IFA, Garbsen: PZH. 